Vol. 42 (1990) [455-464]

\title{
NONOSCILLATION CRITERIA FOR EMDEN-FOWLER SYSTEMS
}

\section{LyNn H. ERBe and XINZHI LiU}

A number of nonoscillation criteria for the Emden-Fowler system

$$
\left\{\begin{array}{l}
x^{\prime}=a(t)|y|^{r_{1}} \operatorname{sgn} y, \\
y^{\prime}=-b(t)|x|^{r_{2}} \operatorname{sgn} x,
\end{array}\right.
$$

where $a(t), b(t)>0, r_{1}, r_{2}>0$ and $b(t) / a(t)$ is locally of bounded variation, are established employing energy function techniques. The results obtained here include many known nonoscillation theorems for the classical Emden-Fowler equations as special cases. We illustrate the results obtained with several examples.

\section{INTRODUCTION}

In this paper we consider the Emden-Fowler system

$$
\left\{\begin{array}{l}
x^{\prime}=a(t)|y|^{r_{1}} \operatorname{sgn} y, \\
y^{\prime}=-b(t)|x|^{r_{2}} \operatorname{sgn} x,
\end{array}\right.
$$

where $a(t)>0, b(t)>0$ are continuous, $r_{1}, r_{2}>0$. A solution of (1.1) is said to be continuable if it exists on the whole half-infinite interval $\left[t_{0}, \infty\right)$. For simplicity, we assume that for any initial value $\left(x_{0}, y_{0}\right)$ the solution of $(1.1)$ is continuable. Conditions which guarantee this may be found, for example, in [5]. A continuable solution is said to be oscillatory if $x(t)$ (and therefore $y(t)$ ) has an infinite number of zeros with $\infty$ as the only accumulation point. The system (1.1) is said to be oscillatory if every pair of continuable solutions $(x(t), y(t))$ is oscillatory and to be nonoscillatory otherwise. In the latter case $x(t)$ and $y(t)$ have constant sign for all large $t$.

When $r_{1}=1$ and $a(t) \equiv 1$, the system (1.1) reduces to the classical Emden-Fowler equation

$$
x^{\prime \prime}+b(t)|x|^{r} \operatorname{sgn} x=0
$$

which has been the object of extensive investigations and we refer to the survey papers $[3,12,13]$ and the references therein for a discussion of oscillatory and nonoscillatory

Received 9th January 1990.

Research supported by NSERC Canada

Copyright Clearance Centre, Inc. Serial-fee code: 0004-9729/90 \$A2.00+0.00. 
properties in this case. Mirzov $[9,10,11]$ generalised many of the well-known oscillation criteria for (1.2) to cover (1.1) and recently Kwong and Wong [7] have extended the well-known oscillation criteria of Atkinson, Belohorec, and Waltman to a more general nonlinear system. We are concerned, in this paper, with obtaining nonoscillation criteria for the Emden-Fowler system (1.1). These include and extend some earlier results of Gollwitzer [6] and the first author [2, 3, 4]. More precisely, it was shown by Atkinson [1] that if $r>1$ (the so-called superlinear case) then all solutions of (1.2) are nonoscillatory if $b(t)$ is nonincreasing and $\int_{t_{0}}^{\infty} t^{r} b(t) d t<\infty$. To replace the nonincreasing assumption on $b(t)$, Gollwitzer [6] introduced the condition $\int_{t_{0}}^{\infty} d b_{+}(t) / b(t)<\infty$ (where the integral is an improper Riemann-Stieltjes integral). Later, it was proved by the first author that $\int_{t_{0}}^{\infty} d b_{+}(t) / b(t)=+\infty$ is compatible with nonoscillations and thus the restriction $\int_{t_{0}}^{\infty} d b_{+}(t) / b(t)<\infty$ was removed. This result has been extended recently by the first author and Lu to the generalised Emden-Fowler differential equation

$$
\left(p(t) x^{\prime}\right)^{\prime}+b(t) x^{r}=0 .
$$

We shall suppose here that the quotient function $b(t) / a(t)$ in system (1.1) is locally of bounded variation on $\left[t_{0}, \infty\right)$ and we set $q(t)=b(t) / a(t)$. We may then write

$$
q(t)=q_{+}(t)-q_{-}(t)
$$

as the Jordan decomposition of $q(t)$ where $q_{+}(t)$ and $q_{-}(t)$ are nondecreasing. We next introduce the notation

$$
Q_{+}(t)=\exp \left(\int_{t_{0}}^{t} \frac{d q_{+}(s)}{q(s)}\right), \quad Q_{-}(t)=\exp \left(\int_{t_{0}}^{t} \frac{d q_{-}(s)}{q(s)}\right)
$$

and clearly both $Q_{+}(t)$ and $Q_{-}(t)$ are nondecreasing and

$$
\frac{Q_{+}(t)}{Q_{-}(t)}=\frac{q(t)}{q\left(t_{0}\right)}
$$

\section{Statement of the Results}

For convenience we first state our main results in this section.

THEOREM 2.1. Let $r_{1} r_{2}>1$. Then the system (1.1) is nonoscillatory if the following condition holds:

$$
\begin{gathered}
\int_{t_{0}}^{\infty}\left(\int_{t_{0}}^{s} a(\tau) d \tau\right)^{r_{2}} b(s) d s<\infty \quad \text { and } \\
\lim _{t \rightarrow \infty}\left(Q_{+}(t)\right)^{\frac{r_{1} r_{2}-1}{r_{1}+1}} \int_{t}^{\infty}\left(\int_{t}^{s} a(\tau) d \tau\right)^{r_{2}} b(s) d s=0 .
\end{gathered}
$$


THEOREM 2.2. Let $r_{1} r_{2}<1$. Then the system (1.1) is nonoscillatory if either one of the following equivalent conditions hold:

$$
\begin{gathered}
\int_{t_{0}}^{\infty}\left(\int_{t_{0}}^{s} a(\tau) d \tau\right)^{r_{2}} b(s) d s<\infty \quad \text { and } \\
\lim _{t \rightarrow \infty}\left(Q_{-}(t)\right)^{\frac{1-r_{1}+r_{2}}{r_{1}+1}} \int_{t}^{\infty}\left(\int_{t}^{s} a(\tau) d \tau\right)^{r_{2}} b(s) d s=0 .
\end{gathered}
$$

REMARK. If $r_{1}=1$ and $a(t)=1 / r(t)$, then Theorems 2.1 and 2.2 include and extend results of $[1,2,4,6]$.

In the half linear case, that is, $r_{1} r_{2}=1$, we have the following neater condition.

COROLLARY 2.3. Let $r_{1} r_{2}=1$. Then the system (1.1) is nonoscillatory if

$$
\int_{t_{0}}^{\infty}\left(\int_{t_{0}}^{s} a(\tau) d \tau\right)^{r_{2}} b(s) d s<\infty
$$

Furthermore, if $Q_{+}(t), Q_{-}(t)$ are bounded above (that is, if $b(t) / a(t)$ is bounded above and below) then (2.3) implies that (1.1) is nonoscillatory for all $r_{1}, r_{2}>0$.

THEOREM 2.4. Let $r_{1} r_{2} \geqslant 1$. Then the system (1.1) is nonoscillatory if

$$
\int_{t_{0}}^{\infty}(b(s))^{\frac{1}{r_{2}+1}}\left(a(s) Q_{+}(s)\right)^{\frac{r_{2}}{r_{2}+1}} d s<\infty
$$

THEOREM 2.5. Let $r_{1} r_{2}<1$. Then the system (1.1) is nonoscillatory if the following condition holds:

$$
\begin{gathered}
\int_{t_{0}}^{\infty}(b(s))^{\frac{1}{r_{2}+1}}\left(a(s) Q_{+}(s)\right)^{\frac{r_{2}}{r_{2}+1}} d s<\infty \quad \text { and } \\
\lim _{t \rightarrow \infty}\left(Q_{+}(t)\right)^{\frac{1-2 r_{1} r_{2}-r_{2}}{\left(r_{1}+1\right)\left(r_{2}+1\right)}}(q(t))^{\frac{r_{1} r_{2}-1}{\left.r_{1}+1\right)\left(r_{2}+1\right)}} \int_{t}^{\infty}(b(s))^{\frac{1}{r_{2}+1}}\left(a(s) Q_{+}(s)\right)^{\frac{r_{2}}{r_{2}+1}} d s=0 .
\end{gathered}
$$

REMARK. Theorems 2.4 and 2.5 extend results of $[2,4,6]$ in the cases $r_{1}=1$ and $a(t) \equiv 1$. The next several results provide further extensions to some of the earlier nonoscillation criteria for equation (1.2).

THEOREM 2.6. Let $r_{1} r_{2}>1$. Then the system (1.1) is nonoscillatory if the following condition holds:

$$
\begin{gathered}
\int_{t_{0}}^{\infty} b(s)\left(Q_{-}(s)\right)^{\frac{r_{2}}{r_{2}+1}} d s<\infty \quad \text { and } \\
\lim _{t \rightarrow \infty}(q(t))^{-\frac{1}{r_{1}+1}} \int_{t}^{\infty} b(s)\left(Q_{-}(s)\right)^{\frac{r_{2}}{r_{2}+1}} d s=0 .
\end{gathered}
$$


Corollary 2.7. Let $r>1$. Then the equation (1.2) is nonoscillatory if the following condition holds:

$$
\begin{gathered}
\int_{t_{0}}^{\infty} b(s)\left(Q_{-}(s)\right)^{\frac{r}{r+1}} d s<0 \text { and } \\
\lim _{t \rightarrow \infty}(q(t))^{-1 / 2} \int_{t}^{\infty} b(s)\left(Q_{-}(s)\right)^{\frac{r}{r+1}} d s=0 .
\end{gathered}
$$

Corollary 2.8. The equation

$$
\left(\frac{x^{\prime}}{a(t)}\right)^{\prime}+b(t) x=0
$$

is nonoscillatory if

$$
\begin{gathered}
\int_{t_{0}}^{\infty} b(s)\left(Q_{-}(s)\right)^{1 / 2} d s<\infty \text { and } \\
\lim _{t \rightarrow \infty}(q(t))^{-1 / 2} \int_{t}^{\infty} b(s)\left(Q_{-}(s)\right)^{1 / 2} d s=0 .
\end{gathered}
$$

TheOREM 2.9. Let $r_{1} r_{2}<1$. Then the system (1.1) is nonoscillatory if the following condition holds:

$$
\begin{gathered}
\int_{t_{0}}^{\infty} b(s)\left(Q_{-}(s)\right)^{\frac{r_{2}}{r_{2}+1}} d s<\infty \text { and } \\
\lim _{t \rightarrow \infty}\left(Q_{-}(t)\right)^{\frac{1-2 r_{1} r_{2}-r_{2}}{\left(r_{1}+1\right)\left(r_{2}+1\right)}}(q(t))^{-\frac{r_{2}}{r_{2}+1}} \int_{t}^{\infty} b(s)\left(Q_{-}(s)\right)^{\frac{r_{2}}{r_{2}+1}} d s=0 .
\end{gathered}
$$

COROLLary 2.10. Let $r<1$. Then the equation (1.2) is nonoscillatory if the following condition holds:

$$
\begin{gathered}
\int_{t_{0}}^{\infty} b(s)\left(Q_{-}(s)\right)^{\frac{r}{r+1}} d s<\infty \quad \text { and } \\
\lim _{t \rightarrow \infty}\left(Q_{-}(t)\right)^{\frac{1-3 r}{2(r+1)}}(q(t))^{\frac{r}{r+1}} \int_{t}^{\infty} b(s)\left(Q_{-}(s)\right)^{\frac{r}{r+1}} d s=0 .
\end{gathered}
$$

To conclude this section, we discuss a few examples below.

Example 1. Consider equation

$$
x^{\prime \prime}+5 t^{-3 / 2}\left(x^{\prime}\right)^{4 / 5} x^{1 / 3}=0, \quad t \geqslant t_{0}>0 .
$$


Letting $y=\left(x^{\prime}\right)^{1 / 5},(2.11)$ becomes the following equivalent Emden-Fowler system

$$
\left\{\begin{array}{l}
x^{\prime}=y^{5} \\
y^{\prime}=-t^{-3 / 2} x^{1 / 3}, \quad t \geqslant t_{0}>0 .
\end{array}\right.
$$

Comparing (2.12) with (1.1), it is easy to see that $a(t) \equiv 1, b(t)=t^{-3 / 2}$, $Q_{+}(t) \equiv 1, r_{1}=5, r_{2}=1 / 3$ and $r_{1} r_{2}=5 / 3>1$. Since

$$
\int_{t_{0}}^{\infty}\left(s-t_{0}\right)^{1 / 3} s^{-s / 2} d s<\infty
$$

it follows from Theorem 2.1 that (2.12) is nonoscillatory.

EXAMPLE 2. Consider equation

$$
x^{\prime \prime}+\frac{5}{3} t^{-12 / 7}\left(x^{\prime}\right)^{2 / 3} x^{3 / 7}=0, \quad t \geqslant t_{0}>0 .
$$

Setting $y=\left(x^{\prime}\right)^{3 / 5},(2.13)$ becomes

$$
\left\{\begin{array}{l}
x^{\prime}=y^{5 / 3} \\
y^{\prime}=-t^{-12 / 7} x^{3 / 7}, \quad t \geqslant t_{0}>0 .
\end{array}\right.
$$

Comparing (2.14) with (1.1), we see that $a(t) \equiv 1, b(t)=t^{-12 / 7}, Q_{-}(t)=t^{12 / 7} / t_{0}^{12 / 7}$, $r_{1}=5 / 3, r_{2}=3 / 7$ and $r_{1} r_{2}=5 / 7<1$. Since

$$
\int_{t_{0}}^{\infty}\left(s-t_{0}\right)^{3 / 7} s^{-12 / 7} d s<\infty
$$

and

$$
\lim _{t \rightarrow \infty}\left(\frac{t^{\frac{12}{7}}}{t_{0}^{\frac{17}{7}}}\right)^{\frac{\left(1-\frac{5}{t}\right)}{\left(\frac{3}{3}+1\right)}} \int_{t}^{\infty}(s-t)^{3 / 7} s^{-12 / 7} d s \leqslant \lim _{t \rightarrow \infty} \frac{7}{2} t_{0}^{-9 / 49} t^{9 / 49} \cdot t^{-2 / 7}=0,
$$

it follows from Theorem 2.2 that (2.14) is nonoscillatory.

EXAMPle 3. Consider equation

$$
\frac{5}{3}\left(x^{\prime}\right)^{2 / 3} x^{\prime \prime}+t^{-11 / 4} x^{5 / 3}=0, \quad t \geqslant t_{0}>0 .
$$

Letting $y=\left(x^{\prime}\right)^{5 / 3}$, we then obtain from (2.15) the following equivalent Emden-Fowler system

$$
\left\{\begin{array}{l}
x^{\prime}=y^{3 / 5}, \\
y^{\prime}=-t^{-11 / 4} x^{5 / 3}, \quad t \geqslant t_{0}>0 .
\end{array}\right.
$$

Comparing (2.16) with (1.1), we see that $a(t) \equiv 1, b(t)=t^{-(11 / 4)}, r_{1}=3 / 5, r_{2}=5 / 3$ and $r_{1} r_{2}=1$. Since

$$
\int_{t_{0}}^{\infty}\left(s-t_{0}\right)^{5 / 3} s^{-11 / 4} d s<\infty
$$

it follows from Corollary 2.3 that (2.16) is nonoscillatory. 


\section{Proof of the Results}

We shall need the following lemma in the proof.

Lemma 3.1. Let $(x(t), y(t))$ be a solution of (1.1). Then for $t \geqslant \zeta \geqslant t_{0}$ we have

$$
\frac{Q_{-}(\zeta)}{Q_{-}(t)} \leqslant \frac{E(t)}{E(\zeta)} \leqslant \frac{Q_{+}(t)}{Q_{+}(\zeta)}
$$

and

$$
\frac{Q_{-}(\zeta)}{Q_{-}(t)} \leqslant \frac{B(\zeta)}{B(t)} \leqslant \frac{Q_{+}(t)}{Q_{+}(\zeta)}
$$

where

$$
E(t)=q(t) \frac{|x|^{r_{2}+1}}{r_{2}+1}+\frac{|y|^{r_{1}+1}}{r_{1}+1}
$$

and

$$
B(t)=\frac{E(t)}{q(t)} .
$$

We omit the proof of the lemma since it is similar to the proof of Lemma 3.1 in [2].

Proof of Theorem 2.1: We suppose, for the sake of contradiction, that $(x(t), y(t))$ is an oscillatory solution of (1.1) and let $t_{2 n} \rightarrow \infty$ be a sequence with $x\left(t_{2 n}\right)=0$ and $y\left(t_{2 n}\right)>0$. Let $t_{2 n+1}>t_{2 n}$ be the first zero of $y(t)$ exceeding $t_{2 n}$. Then for $t_{2 n} \leqslant t \leqslant t_{2 n+1}$, we have $y(t)>0$ and $y^{\prime}(t) \leqslant 0$. Integrating the second equation of (1.1), we obtain

$$
\begin{aligned}
y\left(t_{2 n}\right) & =\int_{t_{2 n}}^{t_{2 n+1}} b(s)(x(s))^{r_{2}} d s=\int_{t_{2 n}}^{t_{2 n+1}} b(s)\left(\int_{t_{2 n}}^{s} x^{\prime}(\tau) d \tau\right)^{r_{2}} d s \\
& =\int_{t_{2 n}}^{t_{2 n+1}} b(s)\left(\int_{t_{2 n}}^{s} a(\tau) y^{r_{1}}(\tau) d \tau\right)^{r_{2}} d s \\
& \leqslant\left(y\left(t_{2 n}\right)\right)^{r_{1} r_{2}} \int_{t_{2 n}}^{t_{2 n+1}} b(s)\left(\int_{t_{2 n}}^{s} a(\tau) d \tau\right)^{r_{2}} d s \\
& \leqslant\left(y\left(t_{2 n}\right)\right)^{r_{1} r_{2}} \int_{t_{2 n}}^{t_{2 n+1}}\left(\int_{t_{2 n}}^{s} a(\tau) d \tau\right)^{r_{2}} b(s) d s .
\end{aligned}
$$

Suppose that condition (i) holds.

Let $\zeta=t_{0}$ and $t=t_{2 n}$ in (3.1); we have, for some $C>0$,

$$
\left(y\left(t_{2 n}\right)\right)^{r_{1} r_{2}-1} \leqslant C\left(Q_{+}\left(t_{2 n}\right)\right)^{\frac{r_{1} r_{2}-1}{r_{1}+1}} .
$$

It then follows from (3.3) and (3.4) that

$$
1 \leqslant C\left(Q_{+}\left(t_{2 n}\right)\right)^{\frac{r_{1} r_{2}-1}{r_{1}+1}} \int_{t_{2 n}}^{\infty}\left(\int_{t_{2 n}}^{s} a(\tau) d \tau\right)^{r_{2}} b(s) d s
$$


Since the right-hand side of (3.5) tends to zero as $t_{2 n} \rightarrow \infty$, we have a contradiction. This completes the proof.

Proof of Theorem 2.2: We assume that $(x(t), y(t))$ is an oscillatory solution of (1) and choose a sequence $t_{2 n} \rightarrow \infty$ such that $x\left(t_{2 n}\right)=0$ and $y\left(t_{2 n}\right)>0$. We let $t_{2 n+1}>t_{2 n}$ be the first zero of $y(t)$ exceeding $t_{2 n}$ and obtain, as in the proof of Theorem 2.1,

$$
y\left(t_{2 n}\right) \leqslant\left(y\left(t_{2 n}\right)\right)^{r_{1} r} \int_{t_{2 n}}^{t_{2 n+1}}\left(\int_{t_{2 n}}^{A} a(\tau) d \tau\right)^{r_{2}} b(s) d s
$$

If condition (i) holds, then we let $\zeta=t_{0}$ and $t=t_{2 n}$ in (3.1) and get, for $C>0$,

$$
\left(y\left(t_{2 m}\right)\right)^{1-r_{1} r_{2}} \geqslant C\left(Q_{-}\left(t_{2 n}\right)\right)^{\frac{r_{1} r_{2}-1}{r_{1}+1}} .
$$

This, together with (3.6), implies

$$
1 \leqslant C^{-1}\left(Q_{-}\left(t_{2 n}\right)\right)^{\frac{1-r_{1} r_{2}}{r_{1}+1}} \int_{t_{2 n}}^{\infty}\left(\int_{t_{2 n}}^{s} a(\tau) d \tau\right)^{r_{2}} b(s) d s
$$

which is a contradiction since the right-hand side of (3.7) tends to zero as $t_{2 n} \rightarrow \infty$. Thus the proof of the theorem is completed.

Proof of Theorem 2.4: Suppose again that $(x(t), y(t))$ is an oscillatory solution with $x\left(t_{2 n}\right)=0$ and $y\left(t_{2 n}\right)>0$ as $t_{2 n} \rightarrow \infty$, and let $y\left(t_{2 n+1}\right)=0, t_{2 n+1}>t_{2 n}$, the first zero of $y(t)$ exceeding $t_{2 n}$. From (3.1) with $\zeta=t_{2 n}$ we have, for $t \geqslant t_{2 n}$ and $C_{1}>\mathbf{0}$,

$$
|x(t)|^{r_{2}} \leqslant C_{1}\left(y\left(t_{2 n}\right)\right)^{\frac{r_{2}\left(r_{1}+1\right)}{r_{2}+1}}\left(Q_{+}\left(t_{2 n}\right)\right)^{-\frac{r_{2}}{r_{2}+1}}\left(Q_{+}(t)\right)^{\frac{r_{2}}{r_{2}+1}}(q(t))^{-\frac{r_{2}}{r_{2}+1}}
$$

and so from the second equation of (1.1) we get

$$
y\left(t_{2 n}\right)=\int_{t_{3 n}}^{t_{2 n+1}} b(s)(x(s))^{r_{2}} d s
$$

$$
\leqslant C_{1}\left(y\left(t_{2 n}\right)\right)^{\frac{r_{2}\left(r_{1}+1\right)}{r_{2}+1}}\left(Q_{+}\left(t_{2 n}\right)\right)^{-\frac{r_{2}}{r_{2}+1}} \int_{t_{2 n}}^{t_{2 n+1}}(b(s))^{\frac{1}{r_{2}+1}}\left(a(s) Q_{+}(s)\right)^{\frac{r_{2}}{r_{2}+1}} d s
$$

If $r_{1} r_{2}=1$, then we have

$$
1 \leqslant C_{1}\left(Q_{+}\left(t_{2 n}\right)\right)^{-\frac{r_{3}}{r_{2}+1}} \int_{t_{2 n}}^{\infty} b(s)^{\frac{1}{r_{2}+1}}\left(a(s) Q_{+}(s)\right)^{\frac{r_{2}}{r_{2}+1}} d s
$$


which is a contradiction because the right-hand side of the above inequality tends to zero as $t_{2 n} \rightarrow \infty$. If $r_{1} r_{2}>1$, then from (3.1) with $\zeta=t_{0}, t=t_{2 n}$, we have, for $C_{2}>0$,

$$
\left(y\left(t_{2 n}\right)\right)^{\frac{r_{2} r_{1}-1}{r_{2}+1}} \leqslant C_{2}\left(Q_{+}\left(t_{2 n}\right)\right)^{\frac{r_{2} r_{1}-1}{\left(r_{1}+1\right)\left(r_{2}+1\right)}}
$$

It then follows from (3.9) and (3.10) that

$$
1 \leqslant C_{1} C_{2}\left(Q_{+}\left(t_{2 n}\right)\right)^{\frac{r_{2} r_{1}-1}{\left.r_{1}+1\right)\left(r_{2}+1\right)}}\left(Q_{+}\left(t_{2 n}\right)\right)^{-\frac{r_{2}}{r_{2}+1}} \int_{t_{2 n}}^{\infty}(b(s))^{\frac{1}{r_{2}+1}}\left(a(s) Q_{+}(s)\right)^{\frac{r_{2}}{r_{2}+1}} d s
$$

$$
\leqslant C_{1} C_{2} \int_{t_{2 n}}^{\infty}(b(s))^{\frac{1}{r_{2}+1}}\left(a(s) Q_{+}(s)\right)^{\frac{r_{2}}{r_{2}+1}} d s
$$

since the right-hand side tends to zero as $t_{2 n} \rightarrow \infty$, we have a contradiction. This proves the theorem.

Proof of Theorem 2.5: Let $(x(t), y(t))$ be an oscillatory solution of $(1.1)$ and $t_{2 n} \rightarrow \infty$ be a sequence such that $x\left(t_{2 n}\right)=0$ and $y\left(t_{2 n}\right)>0$, and let $y\left(t_{2 n+1}\right)=$ $0, t_{2 n+1}>t_{2 n}$, be the first zero of $y(t)$ exceeding $t_{2 n}$. As in the proof of Theorem 2.4, we derive, for $C_{1}>0$,

$$
y\left(t_{2 n}\right) \leqslant C_{1}\left(y\left(t_{2 n}\right)\right)^{\frac{r_{2}\left(r_{1}+1\right)}{r_{2}+1}}\left(Q_{+}\left(t_{2 n}\right)\right)^{-\frac{r_{2}}{r_{2}+1}} \int_{t_{2 n}}^{t_{2 n+1}}(b(s))^{\frac{1}{r_{2}+1}}\left(a(s) Q_{+}(s)\right)^{\frac{r_{2}}{r_{2}+1}} d s
$$

which implies

$$
\left(y\left(t_{2 n}\right)\right)^{\frac{2-r_{1} r_{2}}{r_{2}+1}} \leqslant C_{1}\left(Q_{+}\left(t_{2 n}\right)\right)^{-\frac{r_{2}}{r_{2}+1}} \int_{t_{2 n}}^{t_{2 n+1}}(b(s))^{\frac{1}{r_{2}+1}}\left(a(s) Q_{+}(s)\right)^{\frac{r_{2}}{r_{2}+1}} d s .
$$

Letting $\zeta=t_{0}, t=t_{2 n}$ in (3.2), we have for $C_{2}>0$,

$$
\left(y\left(t_{2 n}\right)\right)^{\frac{1-r_{1} r_{2}}{r_{2}+1}} \geqslant C_{2}\left(Q_{+}\left(t_{2 n}\right)\right)^{\frac{r_{1} r_{2}-1}{\left(r_{1}+1\right)\left(r_{2}+1\right)}}\left(q\left(t_{2 n}\right)\right)^{\frac{1-r_{1} r_{2}}{\left(r_{1}+1\right)\left(r_{2}+1\right)}} .
$$

It then follows from (3.12) and (3.13) that

$$
1 \leqslant \frac{C_{1}}{C_{2}}\left(Q_{+}\left(t_{2 n}\right)\right)^{\frac{1-2 r_{1} r_{2}-r_{2}}{\left(r_{1}+1\right)\left(r_{2}+1\right)}}\left(q\left(t_{2 n}\right)\right)^{\frac{r_{1} r_{2}-1}{\left.r_{1}+1\right)\left(r_{2}+1\right)}} \int_{t_{2 n}}^{\infty}(b(s))^{\frac{1}{r_{2}+1}}\left(a(s) Q_{+}(s)\right)^{\frac{r_{2}}{r_{2}+1}} d s
$$

which is a contradiction since the right-hand side of (3.14) tends to zero as $t_{2 n} \rightarrow \infty$. Thus the proof is completed. 
Proof of Theorem 2.6: Assume that $(x(t), y(t))$ is an oscillatory solution of (1.1) and let $t_{2 n} \rightarrow \infty$ be a sequence with $x\left(t_{2 n}\right)=0$ and $y\left(t_{2 n}\right)>0$. Let $t_{2 n+1}>t_{2 n}$ be the first zero of $y(t)$ exceeding $t_{2 n}$. From (3.2) with $\zeta=t_{2 n}$ we have for $t \geqslant t_{2 n}$ and $C_{1}>0$

$$
|x(t)|^{r_{2}} \leqslant C_{1}\left(y\left(t_{2 n}\right)\right)^{\frac{r_{2}\left(r_{1}+1\right)}{r_{2}+1}}\left(Q_{-}\left(t_{2 n}\right)\right)^{-\frac{r_{2}}{r_{2}+1}}\left(Q_{-}(t)\right)^{\frac{r_{2}}{r_{2}+1}} .
$$

Integrating the second equation of (1.1), we derive

$$
y\left(t_{2 n}\right) \leqslant C_{1}\left(y\left(t_{2 n}\right)\right)^{\frac{r_{2}\left(r_{1}+1\right)}{r_{2}+1}}\left(Q_{-}\left(t_{2 n}\right)\right)^{-\frac{r_{2}}{r_{2}+1}} \int_{t_{2 n}}^{t_{2 n+1}} b(s)\left(Q_{-}(s)\right)^{\frac{r_{2}}{r_{2}+1}} d s .
$$

The rest of the proof is similar to that in the proof of Theorem 2.4 and we omit the details here. Thus the proof is complete.

We also omit the proof of Theorem 2.9 since it is similar to that of Theorem 2.5.

\section{REFERENCES}

[1] F.V. Atkinson, 'On second order nonlinear oscillation', Pacific J. Math. 5 (1955), 643-647.

[2] L.H. Erbe, 'Nonoscillation criteria for second order nonlinear differential equations', $J$. Math. Anal. Appl. 108 (1985), 515-527.

[3] L.H. Erbe, 'Oscillation and nonoscillation properties for second order nonlinear differential equations', in Equadiff 82 (Wurzburg, 1982) Lecture Notes in Math., pp. 168-176 (Springer-Verlag, Berlin, Heidelberg, New York, 1983).

[4] L.H. Erbe and Hong Lu, 'Nonoscillation theorems for second order nonlinear differential equations', Funkcial Ekvac. (to appear).

[5] L.H. Erbe and Zhongehso Liang, 'Continuation and uniqueness for generalized Emden-Fowler systems', (preprint).

[6] H.E. Gollwitzer, 'Nonoscillation theorems for nonlinear differential equations', Proc. Amer. Math. Soc. 26 (1970), 78-84.

[7] M.K. Kwong and J.S.W. Wong, 'Oscillation of Emden-Fowler systems', Differential Integral Equations 2 (1988), 133-141.

[8] W. Leighton, 'On self-adjoint differential equations of second order', J. London Math. Soc. 27 (1952), 37-47.

[9] D.D. Mirzov, 'Oscillation of solutions of a system of nonlinear differential equations', Differenstial'nye Uravneniya 9 (1973), 581-583.

[10] D.D. Mirzov, 'The oscillation of solutions of a system of nonlinear differential equations', Mat. Zametki 4 (1974), 571-576.

[11] D.D. Mirzov, 'Oscillation properties of solutions of a nonlinear Emden-Fowler differential system', Differenstial'nye Urauneniya 16 (1980), 1980-1984.

[12] J.S.W. Wong, 'Oscillation theorems for second order nonlinear differential equations', Bull. Inst. Math. Acad. Sinica 3 (1975), 283-309. 
[13] J.S.W. Wong, 'On the generalized Emden-Fowler equation', SIAM Rev 17 (1975), 339-360.

Department of Mathematics

University of Alberta

Edmonton, Alberta

Canada T6G 2G1

Current address:

Department of Applied Mathematics

University of Waterloo

Waterloo Ontario

Canada N2L 3G1 